\title{
FINITE APPROXIMATE CONTROLLABILITY OF HILFER FRACTIONAL SEMILINEAR DIFFERENTIAL EQUATIONS
}

\author{
JINRONG WANG, A. G. IBRAHIM, AND DONAL O'REGAN
}

Received 01 April, 2019

\begin{abstract}
In this paper, we investigate the finite approximate controllability of fractional semilinear differential equations involving the Hilfer derivative. We show that if the linear part is approximate controllable, then under suitable conditions the semilinear system is finite approximate controllable.
\end{abstract}

2010 Mathematics Subject Classification: 26A33; 93B05

Keywords: finite approximate controllability, Hilfer derivative, fractional differential equation

\section{INTRODUCTION}

An important qualitative behaviour of a dynamic system is controllability. The problem of exact controllability or controllability is to show the existence of a control function, which steers the solution of the system from its initial state to the final state, approximate controllability means that a system can be steered to an arbitrary small neighbourhood of the final state, and finite approximate controllability means that this system is approximate controllability and the chosen control function satisfies simultaneously a finite number of constraints.

Fractional differential equations and inclusions are important because of their applications in various fields, such as physics, mechanics and engineering [3,11, 14]. For existence results for fractional differential equations and inclusions involving the Riemann-Liouville or the Caputo derivative we refer the reader to [4,12,23,27,30,32] and the references therein. Note that Hilfer [11] proposed a generalized RiemannLiouville fractional derivative, for short, the Hilfer fractional derivative, which includes the Riemann-Liouville and the Caputo fractional derivatives. Furati et al. [1] established the existence and uniqueness of a global solution in the space of weighted continuous functions for a fractional differential equation involving the Hilfer derivative. Abbas et al. [20] adopted the weakly Picard operators theory to give Ulam

The first author was supported in part by the National Natural Science Foundation of China (11661016), Training Object of High Level and Innovative Talents of Guizhou Province ((2016)4006), Major Research Project of Innovative Group in Guizhou Education Department ([2018]012). 
stability results for Hilfer type fractional differential inclusions. Wang et al. [26] considered a Hilfer-type fractional differential switched inclusion with noninstantaneous impulsive and nonlocal conditions. Gu and Trujillo [9] obtained some sufficient conditions to ensure the existence of mild solutions of evolution equation with the Hilfer fractional derivative. Yang and Wang $[28,29]$ investigated existence of mild solutions of Hilfer evolution equations and the approximate controllability of Hilfer fractional differential inclusions with nonlocal conditions and Du et al. [7] established the approximate controllability of impulsive Hilfer fractional differential inclusions. Kamocki [13] derived an equivalent definition of the Hilfer derivative and showed that such a derivative is very useful in practical applications. Wang et al. $[24,25]$ studied the controllability of Caputo fractional noninstantaneous impulsive differential inclusions without compactness in reflexive Banach spaces.

For works on controllability, approximate controllability, and finite approximate controllability of differential and inclusions we refer the reader to $[2,5,6,8,10,15-$ $19,21,22,31]$. It is worth mentioning that Menezes et al. [19] investigated the finite approximate controllability for the semilinear heat equation, Mahmudov [18] studied the finite approximate controllability of a semilinear evolution equation in a Hilbert space under the assumption that the linear part of the system is approximately controllability and Mahmudov [16] established, under the assumption that the linear part of the system is approximately controllability, the finite approximate controllability of a nonlocal Sobolev-type evolution equation involving the Caputo fractional derivative.

In this paper, we study the finite approximate controllability of the following Hilfer fractional evolution equation:

$$
\left\{\begin{array}{l}
D_{0^{+}}^{\alpha, \beta} x(t)=A x(t)+f(t, x(t))+B u(t), \text { a.e. } t \in(0, b], \\
I_{0^{+}}^{1-\gamma} x\left(0^{+}\right)=x_{0}
\end{array}\right.
$$

where $\frac{1}{2}<\alpha<1,0 \leq \beta \leq 1, \gamma=\alpha+\beta-\alpha \beta, J=[0, b], b>0, D_{0^{+}}^{\alpha, \beta} x(t)$ is the leftsided Hilfer derivative (see [11]) with lower limit at 0 of order $\alpha$ and type $\beta, E$ is a real Hilbert space, $A$ is the infinitesimal generator of a strongly continuous semigroup $T(t), t>0$ and $I_{0^{+}}^{1-\gamma}$ is the left-sided Riemann-Liouville integral of order $1-\gamma$ with lower limit at 0 . Moreover, $f: J \times E \rightarrow E$ is a function, the control function $u$ is given in $U=L^{p}(J, X), p>\frac{1}{\alpha}$ a Banach space of admissible control functions, with $X$ being a real Hilbert space, $B$ is a bounded linear operator from $X$ into $E$ and $x_{0}$ is a fixed point of $E$.

To the best of our knowledge, up to now, no work was reported on finite approximate controllability of fractional differential equation involving the Hilfer derivative, and this is the main aim of this paper. The paper is organized as follows. In Section 2, we collect some background material about multifunctions and fractional calculus to be used later. We introduce a measure of noncompactness on the space of weighted 
continuous functions. In Section 3, we establish the finite approximate controllability of (1.1). Finally, an example is provided to illustrate our results.

\section{PRELIMINARIES AND NOTATION}

Let $J=[0, b], C(J, E)$ be the Banach space of all $E$ valued continuous functions from $J$ to $E$ with the norm $\|x\|_{C(J, E)}=\sup _{t \in J}\|x(t)\|$. For $a \in[0, b)$ and $0 \leq \gamma \leq 1$, consider the weighted spaces of continuous functions

$$
C_{\gamma}(J, E)=\left\{x \in C((a, b], E):(t-a)^{\gamma} x(t) \in C(J, E)\right\} .
$$

Now $C_{\gamma}(J, E)$ is a Banach space with the norm

$$
\|x\|_{C_{\gamma}(J, E)}=\sup _{t \in(a, b]}\left\|(t-a)^{\gamma} x(t)\right\| .
$$

Let $K_{\alpha}(t)=t^{\alpha-1} P_{\alpha}(t), P_{\alpha}(t)=\int_{0}^{\infty} \alpha \theta M_{\alpha}(\theta) T\left(t^{\alpha} \theta\right) d \theta$, and $S_{\alpha, \beta}(t)=I_{0^{+}}^{\beta(1-\alpha)} K_{\alpha}(t)$, $t \geq 0$, where $M_{\mu}(\theta)=\sum_{n=1}^{\infty} \frac{(-\theta)^{n-1}}{(n-1) \Gamma(1-\mu n)}, \mu \in(0,1), \theta \in \mathbb{C}$ satisfying $\int_{0}^{\infty} \theta^{\tau} M_{\mu}(\theta) d \theta=$ $\frac{\Gamma(1+\tau)}{\Gamma(1+\tau \mu)}$ for $\theta \geq 0$.

Definition 1 ([9, Definition 2.13]). Let $f:[0, b] \times E \rightarrow E$ be a suitable function. By a mild solution of

$$
\left\{\begin{array}{l}
D_{a^{+}}^{\alpha, \beta} x(t)=A x(t)+f(t, x(t)), t \in(0, b], \\
I_{0^{+}}^{1-\gamma} x\left(0^{+}\right)=x_{0}
\end{array}\right.
$$

we mean a function $x \in C((0, b], E)$ which satisfies

$$
x(t)=S_{\alpha, \beta}(t) x_{0}+\int_{0}^{t} K_{\alpha}(t-s) f(s, x(s)) d s, t \in(0, b] .
$$

Remark 1. From [9, Remark 2.14], we have (i) $D_{0^{+}}^{\beta(1-\alpha)} S_{\alpha, \beta}(t)=K_{\alpha}(t), t \in(0, b]$; (ii) When $\beta=0$, the fractional equation (2.1) reduces to the classical RiemannLiouville fractional equation which was studied by Zhou et al. [31]. Note $S_{\alpha, 0}(t)=$ $K_{\alpha}(t)=t^{\alpha-1} P_{\alpha}(t)$; (iii) When $\beta=1$, the fractional equation (2.1) reduces to the classical Caputo fractional equation which has studied by Zhou et al. [28]. Note $S_{\alpha, 1}(t)=S_{\alpha}(t)$, where $S_{\alpha}(t)$ is defined in [28].

In the following we collect the properties for $S_{\alpha, \beta}(t)$ and $K_{\alpha}(t)$ (see [9, Propositions 2.15, 2.16]).

We need the following condition.

$\left(A_{1}\right) T(t)$ is continuous for the uniform operator topology for $t>0$, and there is a $M>1$ such that $\sup _{t \geq 0}\|T(t)\| \leq M$.

Lemma 1. If $\left(A_{1}\right)$ holds, then we have

(i) $P_{\alpha}(t)$ is continuous for the uniform operator topology for $t>0$. 
(ii) For any fixed $t>0, S_{\alpha, \beta}(t)$ and $K_{\alpha}(t)$ are linear bounded operators and for any fixed $x \in E$,

$$
\left\|S_{\alpha, \beta}(t) x\right\| \leq \frac{M t^{\gamma-1}}{\Gamma(\gamma)}\|x\|, \gamma=\alpha+\beta-\alpha \beta,
$$

and

$$
\left\|K_{\alpha}(t) x\right\| \leq \frac{M t^{\alpha-1}}{\Gamma(\alpha)}\|x\| .
$$

(iii) $\left\{K_{\alpha}(t): t>0\right\}$ and $\left\{S_{\alpha, \beta}(t): t>0\right\}$ are strongly continuous, which means that, for any $x \in E$ and $0<t_{1}<t_{2} \leq b$, we have $\left\|K_{\alpha}\left(t_{1}\right) x-K_{\alpha}\left(t_{2}\right) x\right\| \rightarrow 0$ and $\left\|S_{\alpha, \beta}\left(t_{1}\right) x-S_{\alpha, \beta}\left(t_{2}\right) x\right\| \rightarrow 0$, as $t_{1} \rightarrow t_{2}$.

(iv) $S_{\alpha, \beta}(t)$ and $K_{\alpha}(t)$ are compact for $t>0$ if $T(t), t>0$ is compact.

In the text we denote the mild solution which corresponds to the control function $u$ by $x(., u)$ and to the value of it at the terminal point $b$ by $x(b, u)$.

Definition 2. Now (1.1) is said to be approximate controllable on $J$ if for every $x_{b} \in E$, and any $\varepsilon>0$, there exists a control function $u_{\varepsilon} \in L^{p}(J, X)$ such that the corresponding mild solution of (1.1), $x\left(., u_{\varepsilon}\right)$ satisfies $\left\|x\left(b, u_{\varepsilon}\right)-x_{b}\right\| \leq \varepsilon$.

Definition 3. Now (1.1) is said to be finite approximate controllable on $J$ if for every $x_{b} \in E$, and any $\varepsilon>0$, there exists a control function $u_{\varepsilon} \in L^{p}(J, X)$ such that the corresponding mild solution of (1.1), $x\left(., u_{\varepsilon}\right)$ satisfies $\left\|x\left(b, u_{\varepsilon}\right)-x_{b}\right\| \leq \varepsilon$ and $\pi_{\Upsilon} x\left(b, u_{\varepsilon}\right)=\pi_{\Upsilon} x_{b}$, where $\Upsilon$ is a given finite dimensional subspace of $E$ and $\pi_{\Upsilon}$ is the orthogonal projection on $\Upsilon$.

\section{Finite APPROXIMATE CONTROLLABILITY FOR (1.1)}

Let $\varepsilon>0$ and $x_{b} \in E$ be a fixed point. In order to investigate the finite approximate controllable for (1.1) we have the following assumptions:

$\left(H_{1}\right) f: J \times E \rightarrow E$ is a continuous and there is a function $\varphi \in L^{p}\left(J, \mathbb{R}^{+}\right), p>\frac{1}{\alpha}$ and a continuous nondecreasing function $\Omega:[0, \infty) \rightarrow[0, \infty)$ such that for every $x \in C_{1-\gamma}(J, E), \| f\left(t, x(t) \| \leq \varphi(t) \Omega\left(t^{1-\gamma}\|x(t)\|\right)\right.$, for $t \in J$ and $\liminf _{n \rightarrow \infty} \frac{\|\Omega(n)\|}{n}=$ $\mathrm{v}<\infty$.

$\left(H_{2}\right)\{T(t): t>0\}$ is compact semigroup.

$\left(H_{3}\right)$ The linear system associated with (1.1) is approximately controllable in $J$.

Remark 2. Similarly to that in $[16,18]$, we can show that the linear system associated by (1.1) is approximate controllable in $J$ if and only if the condition $B^{*} K_{\alpha}^{*}(b-s) z=0$, for all $s \in J$ implies that $z=0$.

To prove our main result we first present some preliminary lemmas. 
Lemma 2. Let $r>0$ and $B(0, r)=\left\{\psi \in C_{1-\gamma}(J, E):\|\psi\|_{C_{1-\gamma}(J, E)} \leq r\right\}$. The function

$$
\begin{aligned}
& h: B(0, r) \rightarrow E, \\
& h(\psi)=x_{b}-S_{\alpha, \beta}(b) x_{0}-\int_{0}^{b} K_{\alpha}(b-s) f(s, \psi(s)) d s,
\end{aligned}
$$

is continuous and maps $B(0, r)$ into a relatively compact subset of $E$.

Proof. Let $\left(\psi_{n}\right)$ be a sequence in $B(0, r)$ such that $\psi_{n} \rightarrow \psi$ in $B(0, r)$. It follows from (3.1) that

$$
\lim _{n \rightarrow \infty}\left\|h\left(\psi_{n}\right)-h(\psi)\right\| \leq \frac{M}{\Gamma(\alpha)} \lim _{n \rightarrow \infty} \int_{0}^{b}\left\|(b-s)^{\alpha-1} f\left(s, \psi_{n}(s)\right)-(b-s)^{\alpha-1} f(s, \psi(s))\right\| d s .
$$

Note that for any $s \in J$,

$$
\begin{aligned}
& \left\|(b-s)^{\alpha-1} f\left(s, \Psi_{n}(s)\right)-(b-s)^{\alpha-1} f(s, \psi(s))\right\| \\
& \leq(b-s)^{\alpha-1} \varphi(s) \Omega\left(t^{1-\gamma} \psi_{n}(s)\right)+(b-s)^{\alpha-1} \varphi(s) \Omega\left(t^{1-\gamma} \psi(s)\right) \\
& \leq 2(b-s)^{\alpha-1} \varphi(s) \Omega(r) \in L^{1}\left(J, \mathbb{R}^{+}\right) .
\end{aligned}
$$

Then the continuity of $f(s,$.$) and the Lebesgue dominated convergence theorem im-$ plies $\lim _{n \rightarrow \infty}\left\|h\left(\psi_{n}\right)-h(\psi)\right\|=0$, which proves the continuity of $h$.

Now we show that $h$ maps $B(0, r)$ into a relatively compact subset of $E$. Let $\sigma \in$ $(0, b)$. From the compactness of $K_{\alpha}(s), s>0$ and the continuity of $f(s,$.$) , the set$ $\left\{\int_{0}^{b-\sigma} K_{\alpha}(b-s) f(s, \psi(s)) d s: \psi \in B(0, r)\right\}$ is relatively compact in $E$. Furthermore, for any $\psi \in B(0, r)$ we have

$$
\begin{aligned}
& \lim _{\sigma \rightarrow 0}\left\|\int_{0}^{b} K_{\alpha}(b-s) f(s, \psi(s)) d s-\int_{0}^{b-\sigma} K_{\alpha}(b-s) f(s, \psi(s)) d s\right\| \\
& \leq \lim _{\sigma \rightarrow 0}\left\|\int_{b-\sigma}^{b} K_{\alpha}(b-s) f(s, \psi(s)) d s\right\| \\
& \leq \lim _{\sigma \rightarrow 0} \frac{M}{\Gamma(\alpha)} \int_{b-\sigma}^{b}(b-s)^{\alpha-1} \varphi(s) \Omega(r) d s \\
& \leq \lim _{\sigma \rightarrow 0} \frac{\Omega(r) M}{\Gamma(\alpha)}\left(\frac{p-1}{\alpha p-1}\right)^{\frac{p-1}{p}} \sigma^{\alpha-\frac{1}{p}}\|\varphi\|_{L^{p}\left(J, \mathbb{R}^{+}\right)}=0 .
\end{aligned}
$$

This means that the set $\left\{\int_{0}^{b} K_{\alpha}(b-s) f(s, \psi(s)) d s: \psi \in B(0, r)\right\}$ is arbitrarily close to a relatively compact set, and hence it is a relatively compact set.

Next we define the functional

$$
\begin{aligned}
& J_{\varepsilon}: E \times C_{1-\gamma}(J, E) \rightarrow \mathbb{R}, \\
& J_{\varepsilon}(z, \psi)=\frac{1}{2} \int_{0}^{b}\left\|B^{*} K_{\alpha}^{*}(b-s) z\right\|^{2} d s+\varepsilon\left\|\left(I-\pi_{\Upsilon}\right) z\right\|-<z, h(\psi)>,
\end{aligned}
$$

where $B^{*}$ and $K_{\alpha}^{*}(b-\cdot)$ are the Hilbert adjoint of $B$ and $K_{\alpha}(b-\cdot)$. 
In the following lemma we prove some properties for the functional $J_{\varepsilon}$.

Lemma 3. For any $\psi \in C_{1-\gamma}(J, E)$ the map $z \rightarrow J_{\varepsilon}(z, \psi)$ is strictly convex, continuous and for any $r>0$,

$$
\lim \inf _{\|z\| \rightarrow \infty} \inf _{\psi \in B(0, r)} \frac{J_{\varepsilon}(z, \psi)}{\|z\|} \geq \varepsilon .
$$

Proof. Clearly $J_{\varepsilon}$ is strictly convex and the map $z \rightarrow J_{\varepsilon}(z, \psi)$ is continuous (see $[16,18])$. Assume that (3.2) is not true. Then there are two sequence $\left(z_{n}\right)$ of $E$ and $\left(\psi_{n}\right)$ of $B(0, r)$ such that $\lim _{n \rightarrow \infty}\left\|z_{n}\right\|=\infty$ and

$$
\lim \inf _{n \rightarrow \infty} \frac{J_{\varepsilon}\left(z_{n}, \psi_{n}\right)}{\left\|z_{n}\right\|}<\varepsilon
$$

Next, for any $n \geq 1$ set $\widetilde{z_{n}}=\frac{z_{n}}{\left\|z_{n}\right\|}$. From the reflexivity of $E$, there is a subsequence, denoted again by $\widetilde{z_{n}}$, which converges weakly to an element $\widetilde{z} \in E$, and hence it follows from the compactness of $K_{\alpha}^{*}(b-s)$ and the continuity of $B$, that

$$
\lim _{n \rightarrow \infty} B^{*} K_{\alpha}^{*}(b-s) \widetilde{z_{n}}=B^{*} K_{\alpha}^{*}(b-s) \widetilde{z} .
$$

Moreover, according to Lemma 2, $h\left(\psi_{n}\right)$ has a convergent subsequence, denoted again by $h\left(\psi_{n}\right)$, which converges strongly to $z_{0}, z_{0} \in E$. Now

$$
\begin{aligned}
\frac{J_{\varepsilon}\left(z_{n}, \psi_{n}\right)}{\left\|z_{n}\right\|} & =\frac{1}{2\left\|z_{n}\right\|} \int_{0}^{b}\left\|B^{*} K_{\alpha}^{*}(b-s) z_{n}\right\|^{2} d s+\varepsilon\left\|\left(I-\pi_{\Upsilon}\right) \frac{z_{n}}{\left\|z_{n}\right\|}\right\|-<\frac{z_{n}}{\left\|z_{n}\right\|}, h\left(\psi_{n}\right)> \\
& =\frac{\left\|z_{n}\right\|}{2} \int_{0}^{b}\left\|B^{*} K_{\alpha}^{*}(b-s) \widetilde{z_{n}}\right\|^{2} d s+\varepsilon\left\|\left(I-\pi_{\Upsilon}\right) \widetilde{z_{n}}\right\|-<\widetilde{z_{n}}, h\left(\psi_{n}\right)>.
\end{aligned}
$$

We distinguish the following two cases:

Case 1. $\liminf _{n \rightarrow \infty} \int_{0}^{b}\left\|B^{*} K_{\alpha}^{*}(b-s) \widetilde{z_{n}}\right\|^{2} d s>0$. When this holds we have $\liminf _{n \rightarrow \infty} \frac{J_{\mathcal{\varepsilon}}\left(z_{n}, \psi_{n}\right)}{\left\|z_{n}\right\|}=\infty$, which contradicts (3.3).

Case 2. $\liminf _{n \rightarrow \infty} \int_{0}^{b}\left\|B^{*} K_{\alpha}^{*}(b-s) \widetilde{z_{n}}\right\|^{2} d s=0$. When this holds we have from (3.4) and Fatou's lemma that

$$
\int_{0}^{b}\left\|B^{*} K_{\alpha}^{*}(b-s) \widetilde{z}\right\|^{2} d s \leq \liminf _{n \rightarrow \infty} \int_{0}^{b}\left\|B^{*} K_{\alpha}^{*}(b-s) \widetilde{z_{n}}\right\|^{2} d s=0 .
$$

This together assumption $\left(H_{3}\right)$ implies that $\widetilde{z}=0$. On the other hand, since $\Upsilon$ is of finite dimensional $\pi_{\Upsilon}$ is compact, we have $\lim _{n \rightarrow \infty} \pi_{\Upsilon} \widetilde{z_{n}}=\pi_{\Upsilon} 0=0$. Therefore, $\lim _{n \rightarrow \infty}\left(I-\pi_{\Upsilon}\right) \widetilde{z_{n}}=\lim _{n \rightarrow \infty} \widetilde{z_{n}}$, which implies that $\lim _{n \rightarrow \infty}\left\|\left(I-\pi_{\Upsilon}\right) \widetilde{z_{n}}\right\|^{2}=$ $\lim _{n \rightarrow \infty}\left\|\widetilde{z_{n}}\right\|^{2}=1$.

Notice that since $h\left(\psi_{n}\right) \rightarrow z_{0}$ strongly and $\widetilde{z_{n}}, \rightarrow 0$ weakly, $\lim _{n \rightarrow \infty}<\widetilde{z_{n}}, h\left(\psi_{n}\right)>=$ 0 . Then $\liminf _{n \rightarrow \infty} \frac{J_{\varepsilon}\left(z_{n}, \psi_{n}\right)}{\left\|z_{n}\right\|}=\varepsilon$, which contradicts (3.3).

Remark 3. According to Lemma 2, for any $\psi \in C_{1-\gamma}(J, E)$ the map $z \rightarrow J_{\varepsilon}(z, \psi)$ is strictly convex and continuous, and hence for any $\psi \in C_{1-\gamma}(J, E)$ the functional 
$J_{\mathcal{E}}(, \psi)$ admits a unique minimum $z_{\varepsilon, \psi}$. Then we can define a map $\Phi_{\varepsilon}: C_{1-\gamma}(J, E) \rightarrow$ $E, \Phi_{\varepsilon}(\psi)=z_{\varepsilon, \psi}$, that is $z_{\varepsilon, \psi}=\min _{y \in E} J_{\varepsilon}(y, \psi)$.

Next, we present some properties for $\Phi_{\varepsilon}$.

Lemma 4. For any $r>0$, there is a positive real number $R(\varepsilon, r)$ such that

$$
\left\|\Phi_{\varepsilon}(\psi)\right\| \leq R(\varepsilon, r), \forall \psi \in B(0, r) .
$$

Moreover $\Phi_{\varepsilon}$ is continuous on $B(0, r)$.

Proof. Now (3.2) implies the existence of a real number $R(\varepsilon, r)$ such that

$$
\|z\|>R(\varepsilon, r) \text { implies } \inf _{\psi \in B(0, r)} \frac{J_{\varepsilon}(z, \psi)}{\|z\|} \geq \frac{\varepsilon}{2} .
$$

From the definition of $\Phi_{\varepsilon}$ it follows that $J_{\varepsilon}\left(\Phi_{\varepsilon}(\psi), \psi\right) \leq J_{\varepsilon}(0, \psi)=0, \forall \psi \in C_{1-\gamma}(J, E)$, and hence

$$
\inf _{\psi \in B(0, r)} \frac{J_{\varepsilon}\left(\Phi_{\varepsilon}(\Psi), \psi\right)}{\|z\|}=0 .
$$

From (3.7) and (3.8) we get (3.6).

To prove the continuity of $\Phi_{\varepsilon}$ on $B(0, r)$, let $\psi_{n} \rightarrow \psi$ in $B(0, r)$. In view of (3.6) the sequence $\Phi_{\varepsilon}\left(\psi_{n}\right)$ is bounded in the Hilbert space $E$ which is reflexive, so $\Phi_{\varepsilon}\left(\psi_{n}\right)$ has a subsequence, denoted again by $\Phi_{\varepsilon}\left(\psi_{n}\right)$, which converges weakly to $z^{*}$. Therefore, from the definitions of $J_{\varepsilon}$ and $\Phi_{\varepsilon}$ we get

$$
J_{\varepsilon}\left(\Phi_{\varepsilon}(\psi), \psi\right) \leq J_{\varepsilon}\left(z^{*}, \psi\right) \leq \lim \inf _{n \rightarrow \infty} J_{\varepsilon}\left(\Phi_{\varepsilon}\left(\psi_{n}\right), \psi_{n}\right) .
$$

and

$$
\lim \sup _{n \rightarrow \infty} J_{\varepsilon}\left(\Phi_{\varepsilon}\left(\psi_{n}\right), \psi_{n}\right) \leq \lim _{n \rightarrow \infty} J_{\varepsilon}\left(z^{*}, \psi_{n}\right)=J_{\varepsilon}\left(z^{*}, \psi\right) .
$$

It follows from (3.9) and (3.10) that $\lim _{n \rightarrow \infty} J_{\mathcal{\varepsilon}}\left(\Phi_{\varepsilon}\left(\psi_{n}\right), \psi_{n}\right)=J_{\varepsilon}\left(z^{*}, \psi\right)$.

This means that $z^{*}$ is a minimum for $J_{\varepsilon}(, \psi)$. By the uniqueness of the minimum we conclude that $\Phi_{\varepsilon}(\psi)=z^{*}$. It remains to show that $\Phi_{\varepsilon}\left(\psi_{n}\right)$ converges strongly to $\Phi_{\varepsilon}(\psi)$. Note that from the facts that $\psi_{n} \rightarrow \psi$ in $B(0, r), \Phi_{\varepsilon}\left(\psi_{n}\right) \rightarrow \Phi_{\varepsilon}(\psi)$ weakly, $K_{\alpha}^{*}(t), t>0$ is compact and $h$ is continuous we get

$$
\begin{gathered}
\lim _{n \rightarrow \infty} J_{\varepsilon}\left(\Phi_{\varepsilon}\left(\psi_{n}\right), \psi_{n}\right)=J_{\varepsilon}\left(\Phi_{\varepsilon}(\psi), \psi\right), \\
\lim _{n \rightarrow \infty} \int_{0}^{b}\left\|B^{*} K_{\alpha}^{*}(b-s) \Phi_{\varepsilon}\left(\psi_{n}\right)\right\|^{2} d s=\int_{0}^{b}\left\|B^{*} K_{\alpha}^{*}(b-s) \Phi_{\varepsilon}(\psi)\right\|^{2} d s, \\
\lim _{n \rightarrow \infty}<\Phi_{\varepsilon}\left(\psi_{n}\right), h\left(\psi_{n}\right)>=<\Phi_{\varepsilon}(\psi), h(\psi)>,
\end{gathered}
$$

and

$$
\left\|\left(I-\pi_{\Upsilon}\right) \psi\right\| \leq \lim _{n \rightarrow \infty}\left\|\left(I-\pi_{\Upsilon}\right) \Phi_{\varepsilon}\left(\psi_{n}\right)\right\| .
$$

Now (3.11), (3.12), (3.13) and (3.14) imply

$$
\left\|\left(I-\pi_{\Upsilon}\right) \psi\right\|=\lim _{n \rightarrow \infty}\left\|\left(I-\pi_{\Upsilon}\right) \Phi_{\varepsilon}\left(\psi_{n}\right)\right\| .
$$


Since $E$ is a Hilbert space then (3.15) with the fact that $\Phi_{\varepsilon}\left(\psi_{n}\right) \rightarrow \Phi_{\varepsilon}(\psi)$ weakly assures us that $\Phi_{\varepsilon}\left(\psi_{n}\right)$ converges strongly to $\Phi_{\varepsilon}(\psi)$.

In the following theorem we investigate the finite approximate controllability of (1.1).

Theorem 1. Assume that $\alpha>\frac{1}{2}$ and conditions $\left(A_{1}\right),\left(H_{1}\right),\left(H_{2}\right)$ and $\left(H_{3}\right)$ hold. Then (1.1) is finite approximately controllable provided that

$$
\begin{gathered}
\lim _{n \rightarrow \infty} \frac{R(\varepsilon, n)}{n}=\sigma, \\
\frac{M \eta v b^{1-\gamma}}{\Gamma(\alpha)}\|\varphi\|_{L^{p}\left(J, \mathbb{R}^{+}\right)}+\frac{M^{2}\|B\|^{2} b^{1-\gamma+\alpha}}{\alpha[\Gamma(\alpha)]^{2}} \sigma<1,
\end{gathered}
$$

where $\eta=b^{\alpha-\frac{1}{p}}\left(\frac{p-1}{p \alpha-1}\right)^{\frac{p-1}{p}}$.

Proof. According to Remark 3, for any $x \in C_{1-\gamma}(J, E)$ there is a unique element $\Phi_{\varepsilon}(x) \in E$ such that $J_{\varepsilon}\left(\Phi_{\varepsilon}(x), x\right) \leq J_{\varepsilon}(z, x), \forall z \in E$, so we can define a function

$$
\begin{aligned}
& N_{\varepsilon}: C_{1-\gamma}(J, E) \rightarrow C_{1-\gamma}(J, E), \\
& \left(N_{\varepsilon} x\right)(t)=S_{\alpha, \beta}(t) x_{0}+\int_{0}^{t} K_{\alpha}(t-s) f(s, x(s)) d s+\int_{0}^{t} K_{\alpha}(t-s) B u_{\varepsilon, x}(s) d s,
\end{aligned}
$$

where

$$
u_{\varepsilon, x}(s)=B^{*} K_{\alpha}^{*}(b-s) \Phi_{\varepsilon}(x) .
$$

We now show that using the control function, defined by (3.18), any fixed point, $x_{\varepsilon}$, for $N_{\varepsilon}$ is a mild solution for (1.1) and satisfies $\left\|x_{\varepsilon}(b)-x_{b}\right\| \leq \varepsilon$ and $\pi_{\Upsilon} x_{\varepsilon}(b)=\pi_{\Upsilon} x_{b}$. In fact, for $t \in(0, b]$

$$
\begin{aligned}
x_{\varepsilon}(t)= & S_{\alpha, \beta}(t) x_{0}+\int_{0}^{t} K_{\alpha}(t-s) f\left(s, x_{\varepsilon}(s)\right) d s \\
& +\int_{0}^{t} K_{\alpha}(t-s) B B^{*} K_{\alpha}^{*}(b-s) \Phi_{\varepsilon}\left(x_{\varepsilon}\right) d s .
\end{aligned}
$$

Let $z \in E$ and $\lambda$ be a nonzero real number. From the definition of $\Phi_{\varepsilon}\left(x_{\varepsilon}\right)$ we get for $t \in(0, b], J_{\varepsilon}\left(\Phi_{\varepsilon}\left(x_{\varepsilon}\right), x_{\varepsilon}\right) \leq J_{\varepsilon}\left(\Phi_{\varepsilon}\left(x_{\varepsilon}\right)+\lambda z, x_{\varepsilon}\right)$.

It follows from the definition of $J_{\varepsilon}$ that

$$
\begin{aligned}
& \frac{1}{2} \int_{0}^{b}\left\|B^{*} K_{\alpha}^{*}(b-s) \Phi_{\varepsilon}\left(x_{\varepsilon}\right)\right\|^{2} d s+\varepsilon\left\|\left(I-\pi_{\Upsilon}\right) \Phi_{\varepsilon}\left(x_{\varepsilon}\right)\right\|-<\Phi_{\varepsilon}\left(x_{\varepsilon}\right), h\left(x_{\varepsilon}\right)> \\
& \leq \frac{1}{2} \int_{0}^{b}\left\|B^{*} K_{\alpha}^{*}(b-s)\left(\Phi_{\varepsilon}\left(x_{\varepsilon}\right)+\lambda z\right)\right\|^{2} d s+\varepsilon\left\|\left(I-\pi_{\Upsilon}\right)\left(\Phi_{\varepsilon}\left(x_{\varepsilon}\right)+\lambda z\right)\right\| \\
& \quad-<\Phi_{\varepsilon}\left(x_{\varepsilon}\right)+\lambda z, h\left(x_{\varepsilon}\right)>.
\end{aligned}
$$

Now

$\lambda<z, h\left(x_{\varepsilon}\right)>\leq \frac{\lambda^{2}}{2} \int_{0}^{b}\left\|B^{*} K_{\alpha}^{*}(b-s) z\right\|^{2} d s$ 


$$
\left.+\lambda \int_{0}^{b}<B^{*} K_{\alpha}^{*}(b-s) \Phi_{\varepsilon}\left(x_{\varepsilon}\right), B^{*} K_{\alpha}^{*}(b-s) z>d s+\varepsilon|\lambda| \|\left(I-\pi_{\Upsilon}\right) z\right) \| .
$$

If $\lambda>0$, then (3.20) becomes

$$
\begin{aligned}
<z, h\left(x_{\varepsilon}\right)>\leq & \frac{\lambda}{2} \int_{0}^{b}\left\|B^{*} K_{\alpha}^{*}(b-s) z\right\|^{2} d s \\
& \left.+\int_{0}^{b}<B^{*} K_{\alpha}^{*}(b-s) \Phi_{\varepsilon}\left(x_{\varepsilon}\right), B^{*} K_{\alpha}^{*}(b-s) z>d s+\varepsilon \|\left(I-\pi_{\Upsilon}\right) z\right) \| .
\end{aligned}
$$

Let $\lambda \rightarrow 0^{+}$and one obtains

$$
\left.-\varepsilon \|\left(I-\pi_{\Upsilon}\right) z\right) \| \leq \int_{0}^{b}<B^{*} K_{\alpha}^{*}(b-s) \Phi_{\varepsilon}\left(x_{\varepsilon}\right), B^{*} K_{\alpha}^{*}(b-s) z>d s-<z, h\left(x_{\varepsilon}\right)>.
$$

If $\lambda<0$, then (3.20) becomes

$$
\begin{aligned}
<z, h\left(x_{\varepsilon}\right)>\geq & \frac{\lambda}{2} \int_{0}^{b}\left\|B^{*} K_{\alpha}^{*}(b-s) z\right\|^{2} d s \\
& \left.+\int_{0}^{b}<B^{*} K_{\alpha}^{*}(b-s) \Phi_{\varepsilon}\left(x_{\varepsilon}\right), B^{*} K_{\alpha}^{*}(b-s) z>d s-\varepsilon \|\left(I-\pi_{\Upsilon}\right) z\right) \| .
\end{aligned}
$$

Let $\lambda \rightarrow 0^{-}$and one obtains

$$
\left.\int_{0}^{b}<B^{*} K_{\alpha}^{*}(b-s) \Phi_{\varepsilon}\left(x_{\varepsilon}\right), B^{*} K_{\alpha}^{*}(b-s) z>d s-<z, h\left(x_{\varepsilon}\right)>\leq \varepsilon \|\left(I-\pi_{\Upsilon}\right) z\right) \| .
$$

Combining (3.21) and (3.22) and we have

$$
\left.\left|\int_{0}^{b}<B^{*} K_{\alpha}^{*}(b-s) \Phi_{\varepsilon}\left(x_{\varepsilon}\right), B^{*} K_{\alpha}^{*}(b-s) z>d s-<z, h\left(x_{\varepsilon}\right)>\right| \leq \varepsilon \|\left(I-\pi_{\Upsilon}\right) z\right) \| .
$$

Notice that $<z, h\left(x_{\varepsilon}\right)>=<h\left(x_{\varepsilon}\right), z>$. Then

$$
\left.\left|\int_{0}^{b}<K_{\alpha}(b-s) B B^{*} K_{\alpha}^{*}(b-s) \Phi_{\varepsilon}\left(x_{\varepsilon}\right), z>d s-<h\left(x_{\varepsilon}\right), z>\right| \leq \varepsilon \|\left(I-\pi_{\Upsilon}\right) z\right) \| .
$$

From (3.23) and (3.1) (the definition of $h$ ) we get

$$
\begin{aligned}
\mid \int_{0}^{b}<K_{\alpha}(b-s) B B^{*} K_{\alpha}^{*}(b-s) & \Phi_{\varepsilon}\left(x_{\varepsilon}\right)+K_{\alpha}(b-s) f\left(s, x_{\varepsilon}(s)\right), z>d s \\
& \left.-<x_{b}, z>+<S_{\alpha, \beta}(b) x_{0}, z>\mid \leq \varepsilon \|\left(I-\pi_{\Upsilon}\right) z\right) \| .
\end{aligned}
$$

This inequality with (3.19) gives

$$
\left.\left|<x_{\varepsilon}(b)-x_{b}, z>\right| \leq \varepsilon \|\left(I-\pi_{\Upsilon}\right) z\right) \|, \forall z \in E .
$$

Observe that $\left.\|\left(I-\pi_{\Upsilon}\right) z\right)\|=\| z-\pi_{\Upsilon} z \|=\inf _{\theta \in \Upsilon} d(z, \theta)$. 
Since $0 \in \Upsilon$ one obtains $\left.\|\left(I-\pi_{\Upsilon}\right) z\right)\|\leq\| z \|$. Hence (3.24) yields $\left\langle x_{\varepsilon}(b)-x_{b}, z>\leq\right.$ $\varepsilon\|z\|, \forall z \in E$.

Now taking $z=x_{\varepsilon}(b)-x_{b}$, one obtains $\left\|x_{\varepsilon}(b)-x_{b}\right\| \leq \varepsilon$.

On the other hand if we take in (3.24) $z$ in $\Upsilon$, then it follows that $\left.<x_{\varepsilon}(b)-x_{b}, z\right\rangle=0, \forall z \in \Upsilon$, which means that $\pi_{\Upsilon} x_{\varepsilon}(b)=\pi_{\Upsilon} x_{b}$.

Therefore the control function, defined by (3.18) is the desired control function, so it is sufficient to show that $N_{\varepsilon}$ has a fixed point. We use Schauder's fixed point theorem and divide the proof into steps.

Step 1. In this step we show that there is a natural number $n_{0}$ such that $N_{\varepsilon}$ maps $B\left(0, n_{0}\right)$ into itself. Suppose the contrary. Then there are two sequence $\left(x_{n}\right)$ and $\left(y_{n}\right)$ in $C_{1-\gamma}(J, E)$ such that $y_{n}=N_{\mathcal{\varepsilon}}\left(x_{n}\right),\left\|x_{n}\right\|_{C_{1-\gamma}(J, E)} \leq n$ and $\left\|y_{n}\right\|_{C_{1-\gamma}(J, E)}>n$. Recall the definition of $N_{\varepsilon}$ and we have

$$
\begin{aligned}
y_{n}(t)= & S_{\alpha, \beta}(t) x_{0}+\int_{0}^{t} K_{\alpha}(t-s) f\left(s, x_{n}(s)\right) d s \\
& +\int_{0}^{t} K_{\alpha}(t-s) B B^{*} K_{\alpha}(b-s) \Phi_{\varepsilon}\left(x_{n}\right)(s) d s, t \in(0, b] .
\end{aligned}
$$

Then, if $t \in(0, b]$, we get from $\left(H_{1}\right),(2.2),(2.3)$ and Hölder's inequality that

$$
\begin{aligned}
t^{1-\gamma}\left\|y_{n}(t)\right\| \leq & t^{1-\gamma}\left\|S_{\alpha, \beta}(t) x_{0}\right\|+\frac{M t^{1-\gamma} \Omega\left(\left\|x_{n}\right\|_{C_{1-\gamma}(J, E)}\right)}{\Gamma(\alpha)} \int_{0}^{t}(t-s)^{\alpha-1} \varphi(s) d s \\
& +\frac{M^{2}\|B\|^{2} b^{1-\gamma}\left\|\Phi_{\varepsilon}\left(x_{n}\right)\right\|}{[\Gamma(\alpha)]^{2}} \int_{0}^{t}(t-s)^{\alpha-1}(b-s)^{\alpha-1} d s \\
\leq & \frac{M}{\Gamma(\gamma)}\left\|x_{0}\right\|+\frac{M b^{1-\gamma}}{\Gamma(\alpha)} \Omega(n)\|\varphi\|_{L^{p}\left(J, \mathbb{R}^{+}\right)} \eta \\
& +\frac{M^{2}\|B\|^{2} b^{1-\gamma}\left\|\Phi_{\varepsilon}\left(x_{n}\right)\right\|}{[\Gamma(\alpha)]^{2}} \int_{0}^{t}(t-s)^{2 \alpha-2} d s .
\end{aligned}
$$

Notice that $t^{1-\gamma}\left\|y_{n}(0)\right\|=\lim _{t \rightarrow 0} t^{1-\gamma}\left\|y_{n}(t)\right\|$. Then, from (3.6) and (3.17) we have

$$
n<\left\|y_{n}\right\|_{C_{1-\gamma}(J, E)} \leq \frac{M}{\Gamma(\gamma)}\left\|x_{0}\right\|+\frac{M b^{1-\gamma}}{\Gamma(\alpha)} \Omega(n)\|\varphi\|_{L^{p}\left(J, \mathbb{R}^{+}\right)} \eta+\frac{M^{2}\|B\|^{2} b^{2 \alpha-\gamma} R(\varepsilon, n)}{(2 \alpha-1)[\Gamma(\alpha)]^{2}} .
$$

Divide both sides by $n$ and let $n \rightarrow \infty$, and we obtain

$$
1 \leq \frac{M \eta v b^{1-\gamma}}{\Gamma(\alpha)}\|\varphi\|_{L^{p}\left(J, \mathbb{R}^{+}\right)}+\frac{M^{2}\|B\|^{2} b^{2 \alpha-\gamma}}{(2 \alpha-1)[\Gamma(\alpha)]^{2}} \sigma
$$

which contradicts (3.17). Thus we deduce that there is a natural number $n_{0}$ such that $N_{\varepsilon}\left(B\left(0, n_{0}\right)\right) \subseteq B\left(0, n_{0}\right)$.

Step 2. $N_{\varepsilon}$ is continuous on $B\left(0, n_{0}\right)$.

Consider a sequence $\left(x_{n}\right)_{n \geq 1}$ in $B\left(0, n_{0}\right)$ with $x_{n} \rightarrow x$ in $B\left(0, n_{0}\right)$ and let $y_{n}=N_{\varepsilon}\left(x_{n}\right)$ and $y=N_{\varepsilon}(x)$. We show that $y_{n} \rightarrow y$ in $C_{1-\gamma}(J, E)$. Recall the definition of $N_{\varepsilon}$ and 
we get for $t \in(0, b]$,

$$
\begin{aligned}
\left\|t^{1-\gamma} y_{n}(t)-t^{1-\gamma} y(t)\right\|= & \frac{M t^{1-\gamma}}{\Gamma(\alpha)} \int_{0}^{t}(t-s)^{\alpha-1} \| f\left(s, x_{n}(s)\right)-f(s, x(s) \| d s \\
& +t^{1-\gamma} \int_{0}^{t} K_{\alpha}(t-s) B B^{*} K_{\alpha}(b-s)\left\|\Phi_{\varepsilon}\left(x_{n}\right)-\Phi_{\varepsilon}\left(x_{n}\right)\right\| d s .
\end{aligned}
$$

Note that for any $t \in J$, and any $s \in(0, t)$,

$$
\begin{aligned}
& \left\|(t-s)^{\alpha-1} f\left(s, x_{n}(s)\right)-(t-s)^{\alpha-1} f(s, x(s))\right\| \\
& \leq(t-s)^{\alpha-1} \varphi(s) \Omega\left(t^{1-\gamma} x_{n}(s)\right)+(t-s)^{\alpha-1} \varphi(s) \Omega\left(t^{1-\gamma} x(s)\right) \\
& \leq 2(t-s)^{\alpha-1} \varphi(s) \Omega\left(n_{0}\right) \in L^{1}\left(J, \mathbb{R}^{+}\right) .
\end{aligned}
$$

Then the continuity of $f(s,$.$) and the Lebesgue dominated convergence theorem$ gives

$$
\lim _{n \rightarrow \infty} \frac{M t^{1-\gamma}}{\Gamma(\alpha)} \int_{0}^{t}(t-s)^{\alpha-1} \| f\left(s, x_{n}(s)\right)-f(s, x(s) \| d s=0 .
$$

Moreover, in view of Lemma 1 , for any $t \in J$, and any $s \in(0, t)$,

$$
\begin{aligned}
\left\|K_{\alpha}(t-s) B B^{*} K_{\alpha}(b-s)\right\| \Phi_{\varepsilon}\left(x_{n}\right)-\Phi_{\varepsilon}\left(x_{n}\right) \| & \leq 2 \frac{M^{2}\|B\|^{2} R\left(\varepsilon, n_{0}\right)}{[\Gamma(\alpha)]^{2}}(t-s)^{\alpha-1}(b-s)^{\alpha-1} \\
& \leq 2 \frac{M^{2}\|B\|^{2} R\left(\varepsilon, n_{0}\right)}{[\Gamma(\alpha)]^{2}}(t-s)^{2 \alpha-2} \in L^{1}\left(J, \mathbb{R}^{+}\right) .
\end{aligned}
$$

Again by Lemma $4, \Phi_{\varepsilon}$ is continuous, and hence by using the Lebesgue dominated convergence theorem we have

$$
\lim _{n \rightarrow \infty} \int_{0}^{t} K_{\alpha}(t-s) B B^{*} K_{\alpha}(b-s)\left\|\Phi_{\varepsilon}\left(x_{n}\right)-\Phi_{\varepsilon}\left(x_{n}\right)\right\| d s=0,
$$

so, $N_{\varepsilon}$ is continuous on $B\left(0, n_{0}\right)$.

Step 3. For any $t \in J$, the set $\left\{N_{\varepsilon}(x): x \in B\left(0, n_{0}\right)\right\}$ is a relatively compact subset in $E$.

Let $t \in J$ be fixed and $\delta \in(0, b)$ be enough small and put

$$
W_{1, \delta}=\left\{\int_{0}^{t-\delta} K_{\alpha}(t-s) f(s, x(s)) d s: x \in B\left(0, n_{0}\right)\right\},
$$

and

$$
W_{2, \delta}=\left\{\int_{0}^{t-\delta} K_{\alpha}(t-s) B u_{\varepsilon, x}(s) d s: x \in B\left(0, n_{0}\right)\right\} .
$$

Notice that for any $x \in B\left(0, n_{0}\right), t \in(0, b]$ and $s \in(0, t-\delta],\|f(s, x(s))\| \leq \varphi(s) \Omega\left(n_{0}\right)$. This inequality with the compactness of $K(\lambda), \lambda>0$, implies that $W_{1, \delta}$ and $W_{2, \delta}$ are relatively compact subset in $E$.

Next,

$$
\left\|\int_{0}^{t} K_{\alpha}(t-s) f(s, x(s)) d s-\int_{0}^{t-\delta} K_{\alpha}(t-s) f(s, x(s)) d s\right\|
$$




$$
\begin{aligned}
& \leq\left\|\int_{t-\delta}^{t} K_{\alpha}(t-s) f(s, x(s)) d s\right\| \\
& \leq \frac{M \Omega\left(n_{0}\right)}{\Gamma(\alpha)} \int_{t-\delta}^{t}(t-s)^{\alpha-1} \varphi(s) d s .
\end{aligned}
$$

The last term tends to zero as $\delta \rightarrow 0$. Hence the set $\left\{\int_{0}^{t} K_{\alpha}(t-s) f(s, x(s)) d s: x \in\right.$ $\left.B\left(0, n_{0}\right)\right\}$ is arbitrarily close to a relatively compact subset in $E$. Similarly,

$$
\begin{aligned}
& \left\|\int_{0}^{t} K_{\alpha}(t-s) B u_{\varepsilon, x}(s) d s-\int_{0}^{t-\delta} K_{\alpha}(t-s) B u_{\varepsilon, x}(s) d s\right\| \\
& \leq\left\|\int_{t-\delta}^{t} K_{\alpha}(t-s) B u_{\varepsilon, x}(s) d s\right\| \\
& \leq \frac{M^{2}\|B\|^{2} R\left(\varepsilon, n_{0}\right)}{[\Gamma(\alpha)]^{2}} \int_{t-\delta}^{t}(t-s)^{\alpha-1}(b-s)^{\alpha-1} d s \\
& \leq \frac{M^{2}\|B\|^{2} R\left(\varepsilon, n_{0}\right)}{[\Gamma(\alpha)]^{2}} \int_{t-\delta}^{t}(t-s)^{2 \alpha-2} d s \\
& =\frac{M^{2}\|B\|^{2} R\left(\varepsilon, n_{0}\right)}{[\Gamma(\alpha)]^{2}} \frac{\delta^{2 \alpha-1}}{2 \alpha-1} .
\end{aligned}
$$

Since $\alpha \in\left(\frac{1}{2}, 1\right)$ the last term tends to zero as $\delta \rightarrow 0$. Hence the set $\left\{\int_{0}^{t} K_{\alpha}(t-s) B u_{\varepsilon, x}(s) d s: x \in B\left(0, n_{0}\right)\right\}$ is arbitrarily close to a relatively compact subset in $E$. Hence our claim follows.

Step 3. Set

$$
K=\left\{z \in C(J, E): z(t)=t^{1-\gamma} N_{\varepsilon} x(t), t \in(0, b], z(0)=z\left(0^{+}\right), x \in B\left(0, n_{0}\right)\right\} .
$$

We check that $K$ is equicontinuous.

Let $z \in K$. Then there is $x \in B\left(0, n_{0}\right)$ such that for $t \in(0, b], z(t)=t^{1-\gamma} y(t)$, where

$$
y(t)=S_{\alpha, \beta}(t) x_{0}+\int_{0}^{t} K_{\alpha}(t-s)\left(f(s, x(s))+B u_{\varepsilon, x}(s)\right) d s,
$$

and $z(0)=\lim _{t \rightarrow 0+} t^{1-\gamma} y(t)$. It follows for $t=0, \delta \in(0, b]$ that

$$
\lim _{\delta \rightarrow 0+} z(\boldsymbol{\delta})=\lim _{\delta \rightarrow 0+} \delta^{1-\gamma} y(\delta)=\lim _{t \rightarrow 0+} t^{1-\gamma} y(t)=z(0)
$$

Let $t, t+\delta$ be two points in $(0, b]$. Then

$$
\begin{aligned}
\|z(t+\delta)-z(t)\| \leq & \left\|(t+\delta)^{1-\gamma} S_{\alpha, \beta}(t+\delta) x_{0}-t^{1-\gamma} S_{\alpha, \beta}(t) x_{0}\right\| \\
& +\|(t+\delta)^{1-\gamma} \int_{0}^{t+\delta} K_{\alpha}(t+\delta-s)\left(f(s, x(s))+B u_{\varepsilon, x}(s)\right) d s \\
& -t^{1-\gamma} \int_{0}^{t} K_{\alpha}(t-s)\left(f(s, x(s))+B u_{\varepsilon, x}(s)\right) d s \| \\
\leq & (t+\delta)^{1-\gamma}\left\|S_{\alpha, \beta}(t+\delta)-S_{\alpha, \beta}(t)\right\|\left\|x_{0}\right\|
\end{aligned}
$$




$$
\begin{aligned}
& +\left|(t+\delta)^{1-\gamma}-t^{1-\gamma}\right|\left\|S_{\alpha, \beta}(t) x_{0}\right\| \\
& +\left\|(t+\delta)^{1-\gamma} \int_{t}^{t+\delta} K_{\alpha}(t+\delta-s) f(s, x(s)) d s\right\| \\
& \left.+\| \int_{0}^{t}(t+\delta)^{1-\gamma} K_{\alpha}(t+\delta-s) f(s)-t^{1-\gamma}(t-s)^{\alpha-1} P_{\alpha}(t+\delta-s) f(s, x(s))\right] d s \| \\
& +\left\|\int_{0}^{t}\left[t^{1-\gamma}(t-s)^{\alpha-1} P_{\alpha}(t+\delta-s)-t^{1-\gamma} K_{\alpha}(t-s)\right] f(s, x(s)) d s\right\| \\
& \left.+\|(t+\delta)^{1-\gamma} \int_{t}^{t+\delta} K_{\alpha}(t+\delta-s) B u_{\varepsilon, x}(s)\right) d s \| \\
& +\left\|\int_{0}^{t}\left[(t+\delta)^{1-\gamma} K_{\alpha}(t+\delta-s)-t^{1-\gamma}(t-s)^{\alpha-1} P_{\alpha}(t+\delta-s)\right] B u_{\varepsilon, x}(s) d s\right\| \\
& \left.+\| \int_{0}^{t} t^{1-\gamma}(t-s)^{\alpha-1} P_{\alpha}(t+\delta-s)-t^{1-\gamma} K_{\alpha}(t-s)\right] B u_{\varepsilon, x}(s) d s \| \\
& =\sum_{i=1}^{i=8} I_{i} .
\end{aligned}
$$

In view of Lemma 1 we have

$$
\lim _{\delta \rightarrow 0} I_{1}=\lim _{\delta \rightarrow 0}(t+\delta)^{1-\gamma}\left\|S_{\alpha, \beta}(t+\delta)-S_{\alpha, \beta}(t)\right\|\left\|x_{0}\right\|=0,
$$

and

$$
\begin{aligned}
\lim _{\delta \rightarrow 0} I_{2} & =\lim _{\delta \rightarrow 0}\left|(t+\delta)^{1-\gamma}-t^{1-\gamma}\right|\left\|S_{\alpha, \beta}(t) x_{0}\right\| \\
& \leq \frac{M t^{\gamma-1}}{\Gamma(\gamma)}\left\|x_{0}\right\| \lim _{\delta \rightarrow 0}\left|(t+\delta)^{1-\gamma}-t^{1-\gamma}\right|=0 .
\end{aligned}
$$

Again by Lemma 1, $\left(H_{1}\right)$ and the absolute integral of the Lebesgue integral we get

$$
\begin{aligned}
\lim _{\delta \rightarrow 0} I_{3} & =\lim _{\delta \rightarrow 0}\left\|(t+\delta)^{1-\gamma} \int_{t}^{t+\delta} K_{\alpha}(t+\delta-s) f(s, x(s)) d s\right\| \\
& \leq \frac{M \Omega\left(n_{0}\right)}{\Gamma(\alpha)} \lim _{\delta \rightarrow 0}(t+\delta)^{1-\gamma} \int_{t}^{t+\delta}(t+\delta-s)^{\alpha-1} \varphi(s) d s=0 .
\end{aligned}
$$

Similarly,

$$
\begin{aligned}
\lim _{\delta \rightarrow 0} I_{4} & \left.\leq \lim _{\delta \rightarrow 0} \| \int_{0}^{t}(t+\delta)^{1-\gamma} K_{\alpha}(t+\delta-s) f(s)-t^{1-\gamma}(t-s)^{\alpha-1} P_{\alpha}(t+\delta-s) f(s, x(s))\right] d s \| \\
& =\lim _{\delta \rightarrow 0} \| \int_{0}^{t}(t+\delta)^{1-\gamma}(t+\delta-s)^{\alpha-1} P_{\alpha}(t+\delta-s) f(s, x(s)) \\
& \left.\quad-t^{1-\gamma}(t-s)^{\alpha-1} P_{\alpha}(t+\delta-s) f(s, x(s))\right] d s \| \\
& \leq \frac{M \Omega\left(n_{0}\right)}{\Gamma(\alpha)} \lim _{\delta \rightarrow 0} \int_{0}^{t}\left|(t+\delta)^{1-\gamma}(t+\delta-s)^{\alpha-1}-t^{1-\gamma}(t-s)^{\alpha-1}\right| \varphi(s) d s .
\end{aligned}
$$


Since $\varphi \in L^{p}\left(J, \mathbb{R}^{+}\right), \int_{0}^{t}\left[(t+\delta)^{1-\gamma}(t+\delta-s)^{\alpha-1}-t^{1-\gamma}(t-s)^{\alpha-1}\right] \varphi(s) d s$ exists, then by the Lebesgue dominated convergence theorem, we obtain that $\lim _{\delta \rightarrow 0} I_{4}=0$.

Next,

$$
\begin{aligned}
\lim _{\delta \rightarrow 0} I_{5} & \left.=\lim _{\delta \rightarrow 0} \| \int_{0}^{t} t^{1-\gamma}(t-s)^{\alpha-1} P_{\alpha}(t+\delta-s)-t^{1-\gamma} K_{\alpha}(t-s)\right] f(s, x(s)) d s \| \\
& \left.=\lim _{\delta \rightarrow 0} \| \int_{0}^{t} t^{1-\gamma}(t-s)^{\alpha-1} P_{\alpha}(t+\delta-s)-t^{1-\gamma}(t-s)^{\alpha-1} P_{\alpha}(t-s)\right] f(s, x(s)) d s \| .
\end{aligned}
$$

To find this limit, let $\varepsilon>0$ be enough small. We have

$$
\begin{aligned}
\lim _{\delta \rightarrow 0} I_{5} \leq & \Omega\left(n_{0}\right) t^{1-\gamma} \lim _{\delta \rightarrow 0} \int_{0}^{t-\varepsilon}(t-s)^{\alpha-1} \varphi(s) \sup _{s \in[0, t-\varepsilon]}\left\|P_{\alpha}(t+\delta-s)-P_{\alpha}(t-s)\right\| d s \\
& +\lim _{\delta \rightarrow 0} \int_{t-\varepsilon}^{t} t^{1-\gamma}(t-s)^{\alpha-1}\left\|P_{\alpha}(t+\delta-s) f(s)-P_{\alpha}(t-s) f(s) d s\right\| \\
\leq & \Omega\left(n_{0}\right) t^{1-\gamma} \lim _{\delta \rightarrow 0} \int_{0}^{t-\varepsilon}(t-s)^{\alpha-1} \varphi(s) \sup _{s \in[0, t-\varepsilon]}\left\|P_{\alpha}(t+\delta-s)-P_{\alpha}(t-s)\right\| d s \\
& +\frac{2 M \Omega\left(n_{0}\right)}{\Gamma(\alpha)} \lim _{\delta \rightarrow 0} \int_{t-\varepsilon}^{t} t^{1-\gamma}(t-s)^{\alpha-1} \varphi(s) d s \\
\leq & \Omega\left(n_{0}\right) t^{1-\gamma} \lim _{\delta \rightarrow 0} \int_{0}^{t-\varepsilon}(t-s)^{\alpha-1} \varphi(s) \sup _{s \in[0, t-\varepsilon]}\left\|P_{\alpha}(t+\delta-s)-P_{\alpha}(t-s)\right\| d s \\
& +\frac{2 M \Omega\left(n_{0}\right) t^{1-\gamma}}{\Gamma(\alpha)}\|\varphi\|_{L^{p}\left(J, \mathbb{R}^{+}\right)}\left(\frac{p-1}{\alpha p-1}\right)^{\frac{p-1}{p}} \varepsilon^{\frac{\alpha p-1}{p}} .
\end{aligned}
$$

From Lemma $1, \lim _{\delta \rightarrow 0} \sup _{s \in[0, t-\varepsilon]}\left\|P_{\alpha}(t+\delta-s)-P_{\alpha}(t-s)\right\|=0$, and since $\varphi \in$ $L^{p}\left(J, \mathbb{R}^{+}\right)$, then by the Lebesgue dominated convergence theorem, we obtain that $I_{5} \rightarrow 0$ as $\delta \rightarrow 0$ and $\varepsilon \rightarrow 0$.

For $I_{6}$, it follows from (3.6) that

$$
\begin{aligned}
\lim _{\delta \rightarrow 0} I_{6} & =\lim _{\delta \rightarrow 0}(t+\delta)^{1-\gamma}\left\|\int_{t}^{t+\delta} K_{\alpha}(t+\delta-s) B u_{\varepsilon, x}(s) d s\right\| \\
& \leq \lim _{\delta \rightarrow 0} \frac{M(t+\delta)^{1-\gamma}\|B\|}{\Gamma(\alpha)} \int_{t}^{t+\delta}(t+\delta-s)^{\alpha-1}\left\|B^{*} K_{\alpha}(b-s) \Phi_{\varepsilon}(x)\right\| d s \\
& \leq \lim _{\delta \rightarrow 0} \frac{M^{2}(t+\delta)^{1-\gamma}\|B\|^{2} R\left(\varepsilon, n_{0}\right)}{[\Gamma(\alpha)]^{2}} \int_{t}^{t+\delta}(t+\delta-s)^{\alpha-1}(b-s)^{\alpha-1} d s \\
& \leq \lim _{\delta \rightarrow 0} \frac{M^{2}(t+\delta)^{1-\gamma}\|B\|^{2} R\left(\varepsilon, n_{0}\right)}{[\Gamma(\alpha)]^{2}} \int_{t}^{t+\delta}(t+\delta-s)^{2 \alpha-2} d s=0 .
\end{aligned}
$$

For $I_{7}$ from the Lebesgue dominated convergence theorem we have that

$$
\lim _{\delta \rightarrow 0} I_{7} \leq \lim _{\delta \rightarrow 0}\left\|\int_{0}^{t}\left[(t+\delta)^{1-\gamma} K_{\alpha}(t+\delta-s)-t^{1-\gamma}(t-s)^{\alpha-1} P_{\alpha}(t+\delta-s)\right] B u_{\varepsilon, x} d s\right\|
$$




$$
\begin{aligned}
& \left.=\lim _{\delta \rightarrow 0} \int_{0}^{t}\left|(t+\delta)^{1-\gamma}(t+\delta-s)^{\alpha-1}-t^{1-\gamma}(t-s)^{\alpha-1}\right| \| P_{\alpha}(t+\delta-s) B u_{\varepsilon, x}\right) \| d s \\
& \leq \frac{\|B\| M}{\Gamma(\alpha)} \lim _{\delta \rightarrow 0} \int_{0}^{t}\left|(t+\delta)^{1-\gamma}(t+\delta-s)^{\alpha-1}-t^{1-\gamma}(t-s)^{\alpha-1}\right|\left\|B^{*} K_{\alpha}(b-s) \Phi_{\varepsilon}(x)\right\| d s \\
& \leq \frac{\|B\|^{2} M^{2} R\left(\varepsilon, n_{0}\right)}{[\Gamma(\alpha)]^{2}} \lim _{\delta \rightarrow 0} \int_{0}^{t}\left|(t+\delta)^{1-\gamma}(t+\delta-s)^{\alpha-1}-t^{1-\gamma}(t-s)^{\alpha-1}\right|(b-s)^{\alpha-1} d s \\
& =0 .
\end{aligned}
$$

Next,

$$
\begin{aligned}
\lim _{\delta \rightarrow 0} I_{8}= & \left.\lim _{\delta \rightarrow 0} \| \int_{0}^{t} t^{1-\gamma}(t-s)^{\alpha-1} P_{\alpha}(t+\delta-s)-t^{1-\gamma} K_{\alpha}(t-s)\right] B u_{\varepsilon, x}(s) d s \| \\
\leq & \frac{\|B\|^{2} M R\left(\varepsilon, n_{0}\right)}{\Gamma(\alpha)} \\
& \left.\times \lim _{\delta \rightarrow 0} \| \int_{0}^{t} t^{1-\gamma}(t-s)^{\alpha-1} P_{\alpha}(t+\delta-s)-t^{1-\gamma}(t-s)^{\alpha-1} P_{\alpha}(t-s)\right](b-s)^{\alpha-1} d s \| .
\end{aligned}
$$

To find this limit, let $\varepsilon>0$ be enough small, and one has

$$
\begin{aligned}
\lim _{\delta \rightarrow 0} I_{8} \leq & \frac{\|B\|^{2} M R\left(\varepsilon, n_{0}\right)}{\Gamma(\alpha)} t^{1-\gamma} \lim _{\delta \rightarrow 0} \int_{0}^{t-\varepsilon}(t-s)^{2 \alpha-2} \sup _{s \in[0, t-\varepsilon]}\left\|P_{\alpha}(t+\delta-s)-P_{\alpha}(t-s)\right\| d s \\
& +\frac{\|B\|^{2} M R\left(\varepsilon, n_{0}\right)}{\Gamma(\alpha)} \lim _{\delta \rightarrow 0} \int_{t-\varepsilon}^{t} t^{1-\gamma}(t-s)^{2 \alpha-2}\left\|P_{\alpha}(t+\delta-s)-P_{\alpha}(t-s)\right\| d s \\
\leq & \frac{\|B\|^{2} M R\left(\varepsilon, n_{0}\right)}{\Gamma(\alpha)} t^{1-\gamma} \lim _{\delta \rightarrow 0} \int_{0}^{t-\varepsilon}(t-s)^{2 \alpha-2} \sup _{s \in[0, t-\varepsilon]}\left\|P_{\alpha}(t+\delta-s)-P_{\alpha}(t-s)\right\| d s \\
& +\frac{2 M^{2}\|B\|^{2} R\left(\varepsilon, n_{0}\right)}{[\Gamma(\alpha)]^{2}} \lim _{\delta \rightarrow 0} \int_{t-\varepsilon}^{t} t^{1-\gamma}(t-s)^{2 \alpha-2} d s \\
\leq & \frac{\|B\|^{2} M R\left(\varepsilon, n_{0}\right)}{\Gamma(\alpha)} t^{1-\gamma} \lim _{\delta \rightarrow 0} \int_{0}^{t-\varepsilon}(t-s)^{2 \alpha-2} \sup _{s \in[0, t-\varepsilon]}\left\|P_{\alpha}(t+\delta-s)-P_{\alpha}(t-s)\right\| d s \\
& +\frac{2 M^{2}\|B\|^{2} R\left(\varepsilon, n_{0}\right)}{[\Gamma(\alpha)]^{2}}\left(\frac{p-1}{2 \alpha-p-1}\right)^{\frac{p-1}{p}} \varepsilon^{\frac{2 \alpha p-p-1}{p}} .
\end{aligned}
$$

From Lemma $1, \lim _{\delta \rightarrow 0} \sup _{s \in[0, t-\varepsilon]}\left\|P_{\alpha}(t+\delta-s)-P_{\alpha}(t-s)\right\|=0$, and since $2 \alpha p-p-$ $1>0$, then by the Lebesgue dominated convergence theorem, we obtain that $I_{8} \rightarrow 0$ as $\delta \rightarrow 0$ and $\varepsilon \rightarrow 0$.

As a consequence of Step 1, Step 2, Step 3, and from Schauder's fixed point theorem we see that $N_{\varepsilon}$ has a fixed point.

Remark 4. If the function $f$ is uniformly bounded, then (3.16) and (3.17) hold. In fact, suppose that $\|f(t, z)\| \leq \xi, \forall(t, z) \in J \times E$. Then $v=0$. Moreover, for any $r>0$ and any $\psi \in B(0, r)$ we have

$$
\|h(\psi)\| \leq\left\|x_{b}\right\|+\frac{B b^{\gamma-1}}{\Gamma(\gamma)}\left\|x_{0}\right\|+\frac{M \xi}{\Gamma(\alpha)} \int_{0}^{b}(b-s)^{\alpha-1} d s
$$




$$
\leq\left\|x_{b}\right\|+\frac{B b^{\gamma-1}}{\Gamma(\gamma)}\left\|x_{0}\right\|+\frac{M \xi b^{\alpha}}{\Gamma(\alpha+1)}=\zeta .
$$

Then for any $r>0$, any $\psi \in B(0, r)$ and any $z \in E$,

$$
\left|J_{\varepsilon}(z, \psi)\right| \leq \frac{\|B\|^{2} M^{2}\|z\|^{2}}{2[\Gamma(\alpha)]^{2}} \int_{0}^{b}(b-s)^{2 \alpha-2} d s+\varepsilon\|z\|+\|z\| \zeta .
$$

Therefore, $J_{\varepsilon}(z, \psi)$ takes its minimum at $z=0$, and hence $\Phi_{\varepsilon}(\psi)=0, \forall \psi \in B(0, r)$. Consequently, $R(\varepsilon, r)=0$, for any $r>0$, so (3.16) is satisfied with $\sigma=0$. From this and the fact that $v=0,(3.17)$ holds.

We now give an example to illustrate our theory.

Example 1. Take $\alpha=\frac{2}{3}, 0 \leq \beta \leq 1, \gamma=\alpha+\beta-\alpha \beta, J=[0,1]$ and $E=U=$ $L^{2}[0,1]$. Now $E$ is a separable Hilbert space. Let $B: E \rightarrow E, B=I_{d}$, where $I_{d}$ is the identity operator. Define $A: D(A) \subseteq L^{2}[0,1] \rightarrow L^{2}[0,1]$ by $A x=x_{y y}$, where the domain of $A$ is given by: $D(A)=\left\{x \in L^{2}[0,1]: x, x_{y}\right.$ are absolutely continuous, $\left.x_{y y} \in L^{2}[0,1], x(t, 0)=x(t, 1)=0\right\}$.

Consider the following fractional control system:

$$
\left\{\begin{array}{l}
D_{s_{i}^{+}}^{\frac{2}{3}, \beta} x(t, y)=x_{y y}(t, y)+f(t, x(t, y))+B(u(t)), \text { a.e. } t \in(0, b], \\
I_{0^{+}}^{1-\gamma} x(0, y)=x_{0}
\end{array}\right.
$$

where $u \in L^{2}\left(J, L^{2}(J)\right)$.

Notice that $A$ can be written as $A x=\sum_{n=1}^{\infty} n^{2}<x, x_{n}>x_{n}, x \in D(A)$, where $x_{n}(y)=$ $\sqrt{2} \sin n y, n=1,2, \cdots$ is the orthonormal basis of $E$. Moreover, for any $x \in L^{2}[0,1]$, we have $T(t)(x)=\sum_{n=1}^{\infty} e^{-n^{2} t}<x, x_{n}>x_{n}$.

Clearly, $A$ is the infinitesimal generator of the compact $C_{0}$-semigroup $\{T(t): t \geq 0\}$.

Moreover, for any $x \in E$

$$
\begin{aligned}
P_{\alpha}(1-s) x & =\int_{0}^{\infty} \alpha \theta M_{\alpha}(\theta) T\left((1-s)^{\frac{2}{3}} \theta\right) x d \theta \\
& =\frac{2}{3} \int_{0}^{\infty} \theta M_{\alpha}(\theta) \sum_{n=1}^{\infty} e^{-n^{2} \theta(1-s)^{\frac{2}{3}}}<x, x_{n}>x_{n} d \theta .
\end{aligned}
$$

Consider the Mittag-Leffler function $E_{\frac{2}{3}}(m)=\frac{2}{3} \int_{0}^{\infty} \theta M_{\frac{2}{3}}(\theta) e^{-m \theta} d \theta, m \in \mathbb{R}$. Then

$$
P_{\alpha}(1-s) x=\sum_{n=1}^{\infty} E_{\frac{2}{3}}\left(-n^{2}(1-s)^{\frac{2}{3}}\right)<x, x_{n}>x_{n} .
$$

It follows that

$$
K_{\alpha}(1-s) x=(1-s)^{\alpha-1} \sum_{n=1}^{\infty} E_{\frac{2}{3}}\left(-n^{2}(1-s)^{\frac{2}{3}}\right)<x, x_{n}>x_{n},
$$


which leads to

$$
K_{\alpha}^{*}(1-s) x=(1-s)^{\alpha-1} \sum_{n=1}^{\infty} E_{\frac{2}{3}}\left(-n^{2}(1-s)^{\frac{2}{3}}\right)<x, x_{n}>x_{n} .
$$

Next assume that for some $x \in E, K_{\alpha}^{*}(1-s) x=0, \forall s \in[0,1]$. This means that for any natural number $n, E_{\frac{2}{3}}\left(-n^{2}(1-s)^{\frac{2}{3}}\right)<x, x_{n}>=0, \forall s \in(0,1)$. If $x \neq 0$, then there is a $n_{0} \in \mathbb{N}$ such that $<x, x_{n_{0}}>\neq 0$, and hence $E_{\frac{2}{3}}\left(-n_{0}^{2}(1-s)^{\frac{2}{3}}\right)=0, \forall s \in(0,1)$. Thus $0=\lim _{s \rightarrow 1^{-}} E_{\frac{2}{3}}\left(-n_{0}^{2}(1-s)^{\frac{2}{3}}\right)=E_{\frac{2}{3}}(0)=\frac{1}{\Gamma\left(\frac{2}{3}\right)}$, a contradiction. Therefore $\left(H_{3}\right)$ holds.

Let $f: J \times E \rightarrow E$ be a continuous function satisfying $\left(H_{1}\right)$. Then (3.25) is finite approximate if (3.16) and (3.17) hold.

\section{CONCLUSiON}

Motivated by finite approximate controllability for memory problems in mechanics and engineering, we study finite approximate controllability for a class of Hilfer fractional semilinear evolution equations, which is an extension of the work in [16-18]. We establish sufficient conditions to guarantee that the semilinear problem is finite approximate controllable by requiring that the associated linear system is approximately controllable via semigroup theory. For future research, it is of interest to extend the results to non-instantaneous impulsive evolution equation.

\section{ACKNOWLEDGEMENTS}

The authors thank the referees for their careful reading and comments on the manuscript.

\section{REFERENCES}

[1] K. M. Furati, M. D. Kassi, and N. E. Tatar, " Existence and uniqueness for a problem involving Hilfer fractional derivative." Comput. Math. Appl., vol. 64, pp. 1616-1526, 2012, doi: 10.1016/j.camwa.2012.01.009.

[2] K. Aissani and M. Benchohra, "Controllability of impulsive fractional differential equations with infinite delay." Libertas Math., vol. 34, pp. 1-18, 2014, doi: doi:10.14510/xxxxx.

[3] E. Bajlekova, Fractional evolution equations in Banach spaces. (Ph.D. thesis). Cairo: Eindhoven University of Technology, 2001.

[4] K. Balachandran, S. Kiruthika, and J. J. Trujillo, "On fractional impulsive equations of Sobolev type with nonlocal condition in Banach spaces." Comput. Math. Appl., vol. 62, pp. 1157-1165, 2011, doi: 10.1016/j.camwa.2011.03.031.

[5] K. Balachandran and J. Y. Park, "Controllability of fractional integro-differential systems in Banach spaces." Nonlinear Anal.: Hybrid Sys., vol. 3, pp. 363-367, 2009, doi: 10.1016/j.nahs.2009.01.014.

[6] A. Debbouche and V. Antonov, "Approximate controllability of semilinear Hilfer fractional differential inclusions with impulsive control inclusion conditions in Banach spaces." Chaos Solit. Fract., vol. 102, pp. 140-148, 2017, doi: 10.1016/j.chaos.2017.03.023. 
[7] J. Du, W. Jiang, and A. U. K. Niazi, "Approximate controllability of impulsive Hilfer fractional differential inclusions." J. Nonlinear Sci. Appl., vol. 10, pp. 595-6118, 2017, doi: 10.22436/jnsa.010.02.23.

[8] M. Fečkan, J. Wang, and Y. Zhou, “ Controllability of fractional evolution equations of Sobolov type via characteristic solution operators.” J. Optim. Theory Appl., vol. 156, pp. 79-95, 2013, doi: 10.1007/s10957-012-0174-7.

[9] H. Gu and J. J. Trujillo, " Existence of mild solution for evolution equation with Hilfer fractional derivative.” Appl. Math. Comput., vol. 257, pp. 344-354, 2015, doi: 10.1016/j.amc.2014.10.083.

[10] M. Guo, X. Xue, and R. Li, " Controllability of impulsive evolution inclusions with nonlocal conditions." J. Optim. Theory Appl., vol. 120, pp. 355-374, 2004, doi: 10.1023/B:JOTA.0000015688.53162.eb.

[11] R. Hilfer, Applications of fractional calculus in physics. Cairo: World Scientific, 1999.

[12] A. G. Ibrahim, "Differential equations and inclusions of fractional order with impulse effect in Banach spaces." Bull. Malays. Math. Sci. Soc., vol. 43, pp. 69-109, 2020, doi: 10.1007/s40840018-0665-2.

[13] R. Kamocki, "A new representation formula for the Hilfer fractional derivative and its application.” J. Comput. Appl. Math., vol. 308, pp. 39-45, 2016, doi: 10.1016/j.cam.2016.05.014.

[14] A. A. Kilbas , H. Srivastava , and J. J. Trujillo, Theory and applications of fractional differential equations. Amsterdam: Elsevier, 2006.

[15] J. Liang and H. Yang, " Controllability of fractional integro-differential evolution equation with nonlocal conditions." Appl. Math. Comput., vol. 254, pp. 20-29, 2015, doi: 10.1016/j.amc.2014.12.145.

[16] N. I. Mahmudov, "Finite approximate controllability of evolution equations." Appl. Comput. Math., vol. 16, pp. 159-167, 2017.

[17] N. I. Mahmudov, "Finite approximate controllability of evolution systems via resolvent-like operators." arXiv:1806.06930v1 [math.AP], 2018.

[18] N. I. Mahmudov, "Variational approach to finite approximate controllability of Sobolev-type fractional system.” J. Optim. Theory Appl., vol. 184, pp. 671-686, 2020, doi: 10.1007/s10957-0181255-z.

[19] S. B. Menezes, L. J. De, and L. A. Medeiro, "Finite approximate controllability for semilinear heat equation in noncylinderical domains," Ann. Brezillian Academy Sci., vol. 76, pp. 475-487, 2004, doi: 10.1590/S0001-37652004000300002.

[20] A. P. S. Abbas, M. Benchohra, "Ulam stability for Hilfer type fractional differential inclusions via the weakly Picard operators theory." Frac. Calc. Appl. Anal., vol. 20, pp. 384-398, 2017, doi: 10.1515/fca-2017-0020.

[21] N. K. Tomar and J. Dabas, "Controllability of impulsive fractional order semilinear evolution equations with nonlocal condition," J. Nonlinear Evol. Equ. Appl., vol. 5, pp. 57-67, 2012.

[22] J. Wang, M. Fečkan, and Y. Zhou, "Controllability of impulsive fractional order semilinear evolution equations with nonlocal condition," Dynam. Part. Diff. Equ., vol. 11, pp. 71-87, 2014, doi: 10.4310/DPDE.2011.v8.n4.a3.

[23] J. Wang, A. G. Ibrahim, and M. Fečkan, "Nonlocal impulsive fractional differential inclusions with fractional sectorial operators on Banach spaces." Appl. Math. Comput., vol. 257, pp. 103118, 2015, doi: 10.1016/j.amc.2014.04.093.

[24] J. Wang, A. G. Ibrahim, and Y. Z. M. Fečkan, " Controllability of fractional non-instantaneous impulsive differential inclusions without compactness." IMA J. Math. Cont. Inform., vol. 36, pp. 443-460, 2019, doi: 10.1093/imamci/dnx055.

[25] J. Wang, A. G. Ibrahim, and D. O'Regan, "Controllability of noninstantaneous impulsive semilinear functional differential inclusions without compactness.” Indag. Math., vol. 29, pp. 1362-1392, 2018, doi: 10.1016/j.indag.2018.07.002. 
[26] J. Wang, A. G. Ibrahim, and D. O’Regan, "Hilfer-type fractional differential switched inclusions with noninstantaneous impulsive and nonlocal conditions." Nonlinear Anal.: Model. Contr., vol. 20, pp. 921-941, 2018, doi: 10.15388/NA.2018.6.7.

[27] J. Wang, A. G. Ibrahim, and D. O'Regan, "Topological structure of the solution set for fractional non-instantaneous impulsive evolution inclusions." J. Fixed Point Theory Appl., vol. 20, no. Art.59, pp. 1-25, 2018, doi: 10.1007/s11784-018-0534-5.

[28] M. Yang and Q. Wang, " Approximate controllability of Hilfer fractional differential inclusions with nonlocal conditions.” Math. Math. Appl. Sci., vol. 40, pp. 1126-1138, 2017, doi: 10.1002/mma.4040.

[29] M. Yang and Q. Wang, “ Existence of mild solutions for a class of Hilfer fractional evolution equations with nonlocal condition.” Frac. Calc. Appl. Anal., vol. 21, pp. 1120-1138, 2018, doi: 10.1515/fca-2017-0036.

[30] Y. Zhou, Fractional evolution equations and inclusions. Elsevier\& Academic Press, 2015.

[31] Y. Zhou and F. Jiao, "Nonlocal Cauchy problem for fractional evolution equations," Nonlinear Anal.:RWA , vol. 11, pp. 4465-4475, 20104, doi: 10.1016/j.nonrwa.2010.05.029.

[32] Y. Zhou, L. Zhang, and X. Shen, "Existence of mild solutions for fractional evolution equations." J. Integ. Equ. Appl., vol. 25, pp. 557-585, 2013, doi: 10.1216/JIE-2013-25-4-557.

Authors' addresses

JinRong Wang

Department of Mathematics, Guizhou University, Guiyang, Guizhou 550025, P.R. China, and Qufu Normal University, School of Mathematical Sciences, Qufu, Shandong 273165, P.R. China

E-mail address: jrwangegzu.edu.cn

\section{A. G. Ibrahim}

Faculty of Sciences, Department of Mathematics, King Faisal University, Al-Ahsa 31982 Saudi Arabia

E-mail address: agamal@kfu.edu.sa

Donal O'Regan

School of Mathematics, Statistics and Applied Mathematics, National University of Ireland, Galway Ireland

E-mail address: donal oregan@nuigalway. ie 